\title{
Analogue model of ground ruptures due to groundwater pumping in an aquifer above a bedrock ridge
}

\author{
Mariano Cerca $^{1}$, Dora Carreón-Freyre ${ }^{1}$, and Pietro Teatini ${ }^{2}$ \\ ${ }^{1}$ Laboratorio de Mecánica de Geosistemas, Centro de Geociencias, Universidad Nacional Autónoma de \\ México, Santiago de Querétaro 76230, Mexico \\ ${ }^{2}$ Department of Civil, Architectural and Environmental Engineering, University of Padua, Padua 35131, Italy
}

Correspondence: Mariano Cerca (mcerca@geociencias.unam.mx)

Published: 22 April 2020

\begin{abstract}
We report preliminary results of physical experiments of rupture occurrence and propagation associated with land subsidence driven by groundwater pumping and compare our analogue model results with corresponding previously reported numerical results. In particular, we aim to test the development of tensile stresses above a bedrock ridge that forms the base of an aquifer system. The experiment reproduces the main deformation and ruptures observed in field cases and in the numerical analysis.
\end{abstract}

\section{Introduction}

The inception of ground fractures associated with land subsidence in exploited aquifer systems is an increasing induced hazard (Ye et al., 2019). Understanding of the mechanical processes controlling the formation of ground fractures is needed. Three common geologic configurations encountered in aquifer systems prone to deform and fracture because of extensive groundwater pumping have been recently studied by using an advanced numerical model (Frigo et al., 2019). The three cases correspond to (i) reactivation/displacement of a pre-existing fault, (ii) differential compaction due to variations in thickness of aquifer/aquitard layers constituting the aquifer system and (iii) the development of tensile stresses above a bedrock ridge that forms the base of the aquifer system. In particular, the last case is of interest, since its conceptualization predicts the formation of tensional fractures occurring at the surface in a position above the ridge. Numerical simulations prescribe the rupture localization in vertical a plane following a Mohr-Coulomb plastic behaviour, within an otherwise linear elastic medium. To achieve this goal, these authors first analyse the forces and displacements of an initially continuum medium that indicate the zones prone to fracture. Following this, the vertical plane of rupture is localized and simulated by means of special interface elements.
In this contribution we performed a physical experiment of fracture generation to compare with numerical results and evaluate the limits of physical model interpretation. In this first analysis, we have reproduced and discussed the case of tensile fracture above a bedrock ridge (case iii). Scale analogue experiments of land subsidence are rare (e.g. Cerca et al., 2015) but can help engineers to gain insight into the dynamic evolution of aquifer systems through their exploitation. Physical experiments are made with analogue materials that behave mechanically similar to natural materials in scale. Their similitude with the natural process is assessed in terms of geometry, kinematics and dynamics (Hubbert, 1937). Reproduction of the initial conditions of the natural prototypes, the choice of the materials, the deformation box and the technique to build the model can also have an effect in the results. Comparison with numerical results may be relevant if the evolution of the models is similar. Sensitivity to the frictional behaviour of the granular material used allows for reproducing fractures and faults with geometrical characteristics similar to those observed in natural examples. The results can provide a general confirmation of the possible ruptures developed in numerical models and natural cases. 


\section{Experimental fracturing of aquifer systems}

Physical experiments of aquifer systems were conducted in the Laboratorio de Mecánica de Geosistemas of the Universidad Nacional Autónoma de México (UNAM). Models were built into a plexiglass box with dimensions of $20 \times 20 \times 10 \mathrm{~cm}$. The box has a geometrical arrangement of holes at the base, which are closed during the building of the model. A relatively rigid tabular sponge $1 \mathrm{~cm}$ thick was placed at the bottom of the box in order to prevent the granular material from filling the perforations. For the case presented here, plasticine was used to mould the geometry of the buried rigid bedrock ridge that forms the base of the sedimentary aquifer system. Posteriorly, the box was half filled with water. A fine-grain-sized and well-sorted, slightly cohesive granular medium (glass microbeads) was added until reaching the water level. The microbeads were added loosely using a sieve to maintain an initial high porosity. Subsidence in the experiment is related to the loss of porosity caused by changes in the effective stress produced by the drop of the piezometric level during water extraction at the bottom of the experimental box. Experiments are a simplification of the actual geological setting and thus have limitations: (1) the granular material is relatively homogeneous, contrasting with the heterogeneity observed in natural systems; (2) the granular material has had a frictional behaviour since the beginning of the experiment; and (3) the water table is assumed to be parallel to the surface of the lacustrine sediments at the beginning of the process in the model.

Scaling is made following the procedure explained in Cerca et al. (2015). The dimensionless scaling ratio is of the order of $5.2 \times 10^{-4}$. For simplicity we consider that the differences in the effective stresses are related to the pore pressure drop, which in turn is proportional to the change in the phreatic level. The experimental time for the model was about $2 \mathrm{~min}$. Water extraction was induced by opening the array of holes at the base of the plexiglass box. The deformation at the surface is monitored by means of an optical system allowing for the obtaining of a high-resolution digital elevation model. Digital photographs are recorded at regular intervals $(1 \mathrm{~s})$ from the lateral wall of the plexiglass box to monitor the vertical displacements caused by water extraction.

The photographs obtained from the lateral wall were analysed using a digital image correlation (DIC) technique that permits us to estimate the vertical component of the displacement on a vertical section. DIC relies on the comparison of two images of the lateral wall, which correspond to two different states of deformation (Barrientos et al., 2008). The strain plane state between the reference and displaced images can be obtained by cross correlation. The images are divided into sub-images, generally of the size $32 \times 32$ pixels, and these sub-images are then correlated. DIC was performed using JPIV (https://www.jpiv.vennemann-online.de/

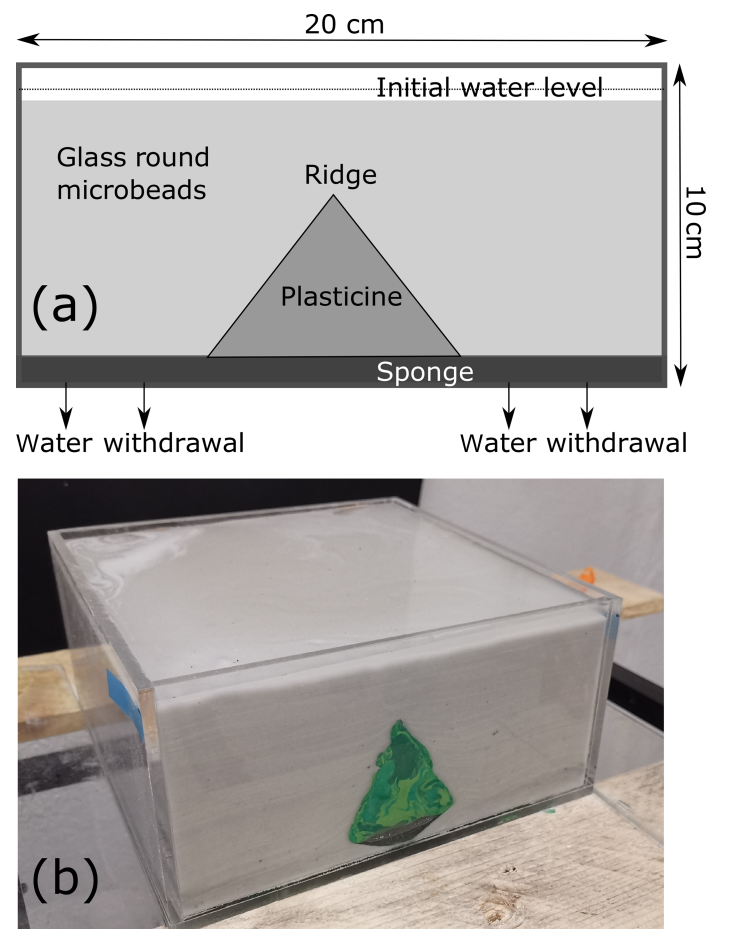

Figure 1. Sketch of the lab experimental setup (a) and photograph of the setup at the start of the experiment (b).

index.html, last access: 18 March 2020), an open-source software package for Particle Image Velocimetry (PIV).

\section{Results and discussion}

The hydromechanical analogue model presented here necessarily simplifies the natural hydrogeological setting. In order to keep the physical experiment simple, we have considered the use of only two materials: a rigid basement and a saturated granular material. In nature, heterogeneity of the sedimentary material can be of importance to the deformation obtained. The force driving the deformation of the compressible material is the pore pressure decrease. Noticeable effects due to differences in water extraction rates were observed but are not considered in the analysis. The main effect observed was the formation of subsidence bowls formed above the location of the basal holes. From the mechanical point of view, wet microbeads form fractures and faults that follow a Mohr-Coulomb behaviour with an angle of friction around $30^{\circ}$ and negligible cohesion. The use of a Mohr-Coulomb criterion to model ground fissures was proposed previously by Budhu (2008). Lateral walls create a border effect that limits the fracture propagation. Because of this boundary effect, DIC performed on the lateral wall might underestimate displacement or create artefacts. This was the case for the experiment showed here.

In spite of the above limitations, the experimental results reproduce ground fractures whose trace in the surface is in- 
(a) Plain view

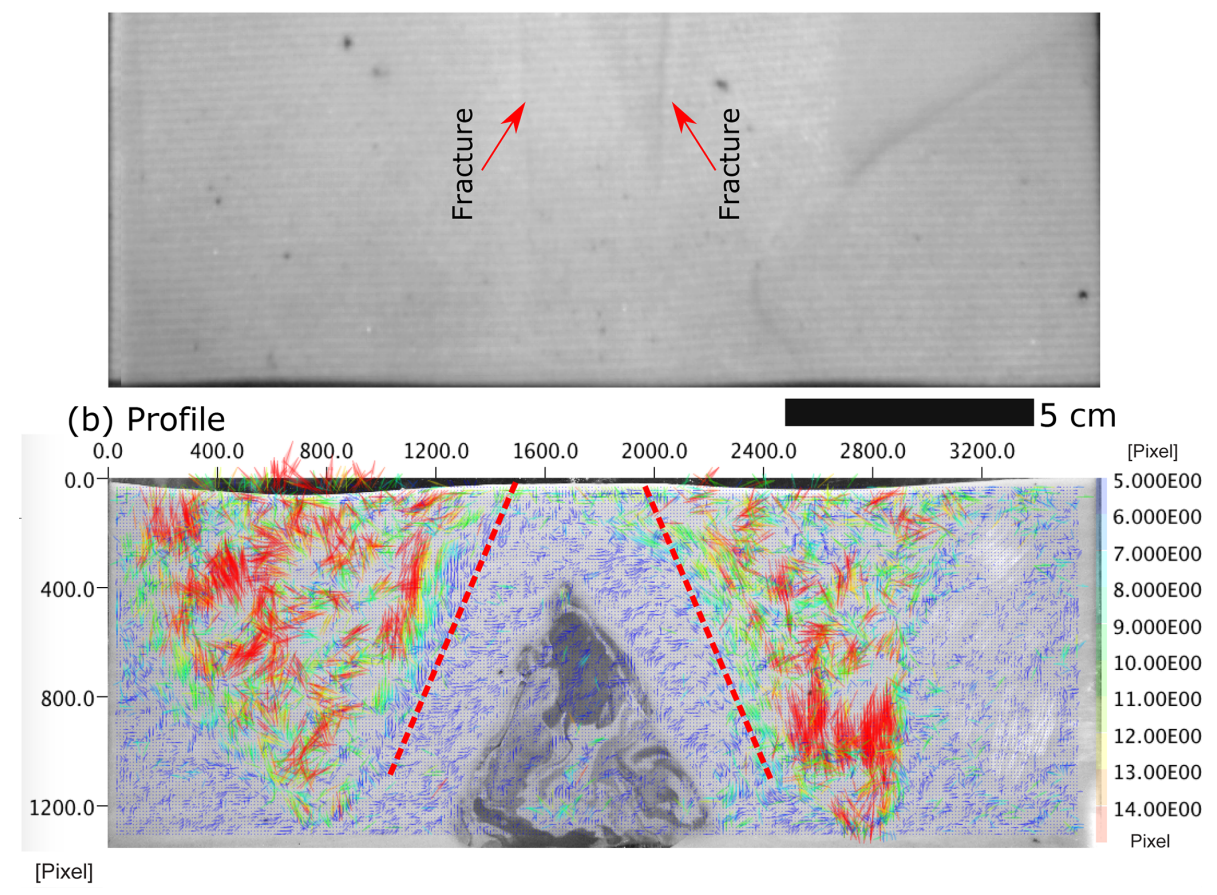

Figure 2. Experimental results: (a) top-view photograph of the experiment showing the formation of fractures at the surface of the model and (b) photograph of the lateral wall of the experiment with the displacements obtained with DIC.

fluenced by the presence of the ridge below. As observed in natural cases, the geometry of the fractures resembles an extensional fault. The inclination of the fractures is of about $67^{\circ}$.

Known cases of fractures formed above a ridge include (Frigo et al., 2019):

a. Casa Grande, in the Picacho Basin, Arizona (Jachens and Holzer, 1982), where using a geophysical survey, these authors showed that ruptures in the alluvial deposits developed in correspondence with local bedrock ridges after intensive groundwater extraction. In this case, the differential deformation resulted in the formation of two faults with opposite inclinations at both sides of the buried ridge, such as in our experimental results.

b. Wuxi in Jiangsu province, China (Ye et al., 2016, 2018), where ground fractures have a banded distribution whose orientation is aligned with the bedrock ridges buried by the Yangtze alluvial deposits. In this case a band of fractures developed with normal fault geometry predominantly dipping northwest. The geometry of this fracture is probably controlled by the asymmetric thickness of the compressible sediments between the two sides of the ridge (larger at the western side; Ye et al., 2018).

Overall our experiment shows that analogue models can successfully simulate the results of previous finite element numerical models and are qualitatively similar to those obtained by Frigo et al. (2019). Dynamic scaling of the frictional material is straightforward, but the geometrical similitude is not completely achieved, since the angle of fracture formation is prescribed as vertical in the numerical model. However, similar to numerical models, water extraction from the bottom of the box produced vertical displacements in the granular material that increase away from the ridge symmetrically (Fig. 2). The displacements near the slopes of the ridge decrease significantly because of the small thickness of the compressible material. The experimental results reproduce also some of the general observations made by Budhu (2008), i.e. the surface of the experiment evolved from (1) gentle bending to (2) rupture and (3) shearing. This contribution shows the potentiality of physical experimentation to describe land subsidence. The results are valuable for validation of physical and numerical models related to the analysis of the dynamic behaviour of deformation associated with groundwater flow in the earth. Land subsidence is a hazard in many exploited aquifers; thus the possibility to foresee its behaviour in a more precise way is extremely important in order to elaborate more rigorous physical models and improve the predictive capacity of the numerical simulators. However, it still can be challenging to reproduce the numerical setups because of the mechanical properties and the friction developing along the box walls. Future work will include variations of selected parameters such as compress- 
ibility of the materials, thickness of the compressible layers, friction of the granular material and geometry of the buried ridge.

Data availability. Data are not publicly available at the moment for this preliminary experiment. They will be available once a complete series of the experiments is finished.

Author contributions. MC made the preliminary experiment and wrote the first draft of the paper. DC and PT provided the general idea to test in the experiments and contributed to writing the paper.

Competing interests. The authors declare that they have no conflict of interest.

Special issue statement. This article is part of the special issue "TISOLS: the Tenth International Symposium On Land Subsidence - living with subsidence". It is a result of the Tenth International Symposium on Land Subsidence, Delft, the Netherlands, 17-21 May 2021.

Acknowledgements. We would like to thank Ricardo Carrizosa, who helped with the experimental setup.

\section{References}

Barrientos, B., Cerca, M., Garcia-Marquez, J., and HernandezBernal, C.: Three-dimensional displacement fields measured in a deforming granular-media surface by combined fringe projection and speckle photography, J Opt A-Pure Appl Op., 10, 104027, https://doi.org/10.1088/1464-4258/10/10/104027, 2008.

Budhu, M.: Mechanisms of earth fissures using the Mohr-Coulomb failure criterion, Environ. Eng. Geosci., 14, 281-295, 2008.

Cerca, M., Rocha, L., Carreón-Freyre, D., and Aranda, J.: Physical experiments of land subsidence within a maar crater: insights for porosity variations and fracture localization, Proc. IAHS, 372, 285-290, https://doi.org/10.5194/piahs-372-285-2015, 2015.

Frigo, M., Ferronato, M., Yu, J., Ye, S., Galloway, D., Carreón-Freyre, D., and Teatini, P.: A parametric numerical analysis of factors controlling ground ruptures caused by groundwater pumping, Water Resour. Res., 55, 9500-9518, https://doi.org/10.1029/ 2019WR025034, 2019.

Hubbert, M. K.: Theory of scale models as applied to the study of geologic structures, Geol. Soc. Am. B., 48, 1459-1520, https://doi.org/10.1130/GSAB-48-1459, 1937.

Jachens, R. C. and Holzer, T. L.: Differential compaction mechanism for earth fissures near Casa Grande, Arizona, Geol. Soc. Am. B., 93, 998-1012, 1982.

Ye, S., Xue, Y., Wu, J., Yan, X., and Yu, J.: Progression and mitigation of land subsidence in China, Hydrogeol. J., 24, 685-693, 2016.

Ye, S., Franceschini, A., Zhang, Y., Janna, C., Gong, X., Yu, J., and Teatini, P.: A novel approach to model earth fissure caused by extensive aquifer exploitation and its application to the Wuxi case, China, Water Resour. Res., 54, 2249-2269, https://doi.org/10.1002/2017WR021872, 2018.

Ye, S., Carreón Freyre, D., Teatini, P., and Galloway, D.: IGCP 641 Project: Mechanisms, Monitoring and Modeling Earth Fissure Generation and Fault Activation due to Subsurface Fluid Exploitation, Acta Geol. Sin.-Engl., 93, 165-168, https://doi.org/10.1111/1755-6724.14279, 2019. 